\title{
Preprocessing of temperature measurement by IR techniques using POD truncated basis for heat source estimation
}

\author{
by N. Ranc*, A. Blanche**, D. Ryckelynck*** and A. Chrysochoos** \\ * ENSAM, Laboratoire PIMM, UMR CNRS 8006, 75013 Paris, France, nicolas.ranc@ensam.eu \\ ** Université Montpellier 2, LMGC, UMR CNRS 5508, 34095 Montpellier, France \\ *** Ecoles des Mines de Paris, Centre des Matéraux, UMR CNRS 7633, 91003 Evry, France
}

\begin{abstract}
Temperature variation induced by the deformation of a solid material is related to heat sources of various natures like energy dissipation or thermo-mechanical couplings. The heat sources are thus interesting data to understand and model the thermo-mechanical behavior of materials. Moreover the infrared thermography allows to measure the temperature fields on a specimen surface but the temperature fields depend on the thermal properties of the material, on the geometry and on the boundary conditions. It is thus necessary to identify heat sources from the temperature measurements. Various techniques exist in the literature but the difficulties mainly come from the noise of the experimental data. In this paper we propose to develop a preprocessing in order to reduce the noise using a decomposition of the measured temperature fields in a truncated basis made of proper orthogonal modes derived from a set of physically admissible thermal fields associated with the considered heat diffusion problem. After this preprocessing, classical methods like finite difference, spectral decomposition or local fitting, are applied and the results are compared.
\end{abstract}

\section{Introduction}

Temperature variation induced by the deformation of a solid material is related to heat sources of various natures like energy dissipation or thermo-mechanical couplings. Dissipation reflects the irreversibility of the deformation process and coupling sources come from an interdependence of the thermal and mechanical properties of the material.

The heat sources are thus interesting data to understand and model the thermomechanical behavior of materials. Moreover the measurement technique of the temperature fields by infrared thermography developed considerably these last years and now allows to carry out measurements with a higher number of pixels, higher acquisition frequencies and with a better signal to noise ratio.

However the temperature variations on the surface of a specimen, during a tensile test for example, do not depend solely on the heat sources associated with the mechanical loading. The temperature fields also depend on the thermal properties of the material (thermal conductivity, heat capacity...), on the geometry of the specimen and of the boundary conditions reflecting the thermal exchanges with the surrounding air and the testing machine.

\section{Heat equation and heat source identification from experimental data}

The link between the temperature variation $\theta(x, y, z, t)$ and the heat sources fields is provided by the heat diffusion equation under certain assumptions [1]:

$$
\rho C \dot{\theta}-k \Delta \theta=d_{1}+s_{t h}=s(x, y, z, t),
$$

with $\rho$ the density, $C$ the specific heat capacity, $k$ the isotropic heat conduction coefficient, $\Delta$ the Laplacian operator, $d_{1}$ the intrinsic dissipation and $s_{\text {th }}$ the heat source associated to thermo-mechanical couplings.

Quantitative IR thermography techniques allow us the measurement of surface temperature fields and thus does not enable to simply calculate the heat sources. In the case of an ultrasonic fatigue test specimen (Fig.1), the temperature variation at the surface of thin, flat specimens is in general supposed to be close to the temperature variations $\vartheta(x, t)$ averaged over the cross section. The tri-dimensional problem of heat diffusion can then be reduced to a one-dimensional problem [2,3]. In this case, the heat diffusion equation can be written as: 


$$
\rho C\left(\frac{\partial \vartheta}{\partial t}+\frac{\vartheta}{\tau_{t h}^{1 D}(x)}\right)-k\left(\frac{S^{\prime}}{S} \cdot \frac{\partial \vartheta}{\partial x}+\frac{\partial^{2} \vartheta}{\partial x^{2}}\right)=s(x, t)
$$

with $S$ the specimen section, $S^{\prime}$ its derivation with respect to $x, \tau_{t h}^{1 D}$ the characteristic time of heat losses due to convection on the specimen surface and $s(x, t)$ the heat source.

The identification of the heat source $s(x, t)$ can be carried out from the estimate of the differential operators with respect to space and to time of the left hand side of Equation (2). However thermal measurements are always noisy and the method to calculate the derivatives may become highly unstable. Various methods were used in the literature in order to limit the noise effects: Gaussian convolutive filtering [4,5], spectral methods [1,6], and local least-squares fitting [2].

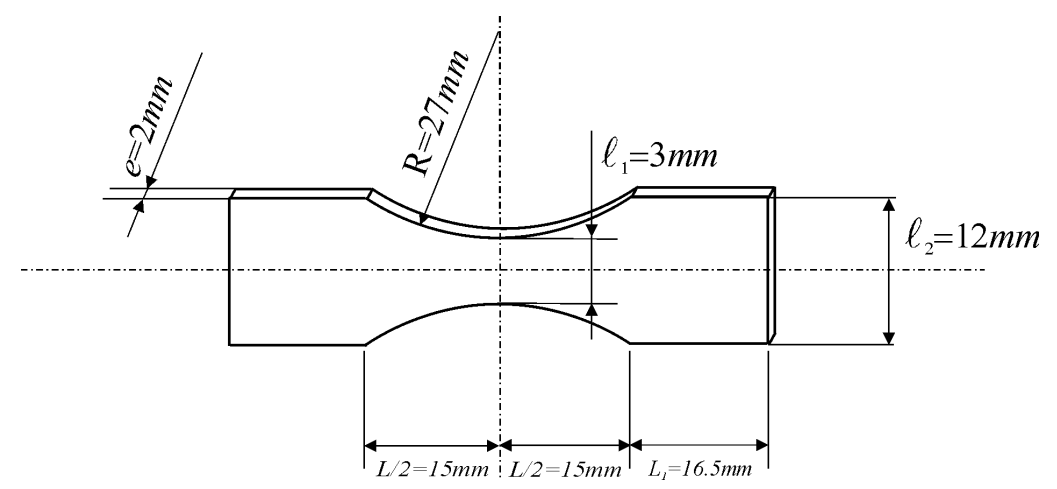

Fig. 1. Geometry of an ultrasonic fatigue test specimen.

\section{Application of POD preprocessing to thermal data}

The aim of this paper is to show that the prior use of reduced-basis projection of thermal data improves the signal-to-noise ratio before estimating the distributions of heat sources. The reduced basis is generated by a proper orthogonal decomposition (POD) of physically-admissible thermal fields [7].

These fields are solutions of ideal diffusion problems, noted $\left(\vartheta^{(r)}\right)_{r=1, \ldots, M}$, related to a set of putative heat sources $\left(s^{(r)}\right)_{r=1, \ldots, M}$ that are time constant point source in space. They span a subspace whose reduced basis is generated by using the POD method. The experimental temperature field is then projected onto the reduced basis and allows to drastically reduce the noise effects:

$$
\vartheta(x, t)=\sum_{k=1}^{N} a_{k}(t) \varphi_{k}(x)
$$

where $\left(\varphi_{k}\right)_{r=1, \ldots, N}$ are the proper orthogonal modes (POMs) of the POD reduced basis. There are solutions of the following equation [7]:

$$
\varphi_{k}=\arg \max _{\psi} \frac{\sum_{r=1}^{M} \int_{0}^{t_{f}}\left(\int_{\Omega} \vartheta^{(r)}(x, t) \psi(x) \mathrm{d} \Omega\right)^{2} \mathrm{~d} t}{\int_{\Omega} \psi^{2}(x) \mathrm{d} \Omega},
$$

where $\Omega$ is the spatial domain and $\left[0, t_{f}\right]$ is the time interval. This equation means that functions $\psi(x)$ which maximize their projection on the calculated temperature fields for every time and in and $\left[0, t_{f}\right]$ are search. There are many solutions to this maximum problem and the POM(s) are ordered such that: 


$$
\sigma_{k}^{2} \geq \sigma_{k+1}^{2}
$$

where $\sigma_{k}^{2}$ is the positive eigenvalue related to the $\mathrm{POM} \varphi_{k}$ defined as :

$$
\sigma_{k}^{2}=\frac{\sum_{r=1}^{M} \int_{0}^{t_{f}}\left(\int_{\Omega} \vartheta^{(r)}(x, t) \varphi_{k}(x) \mathrm{d} \Omega\right)^{2} \mathrm{~d} t}{\int_{\Omega} \varphi_{k}^{2}(x) \mathrm{d} \Omega} .
$$

Fig. 2a represents the eigenvalues of various proper orthogonal modes arranged in decreasing order. These eigenvalues represents the level of energy of each mode and decrease quickly. It is thus possible to truncate this POD basis without affecting the total energy of the system. In our case, a truncated base from approximately 10 to 15 modes will be used. In Fig. $2 b$ the first four modes are plotted according to the space coordinate.

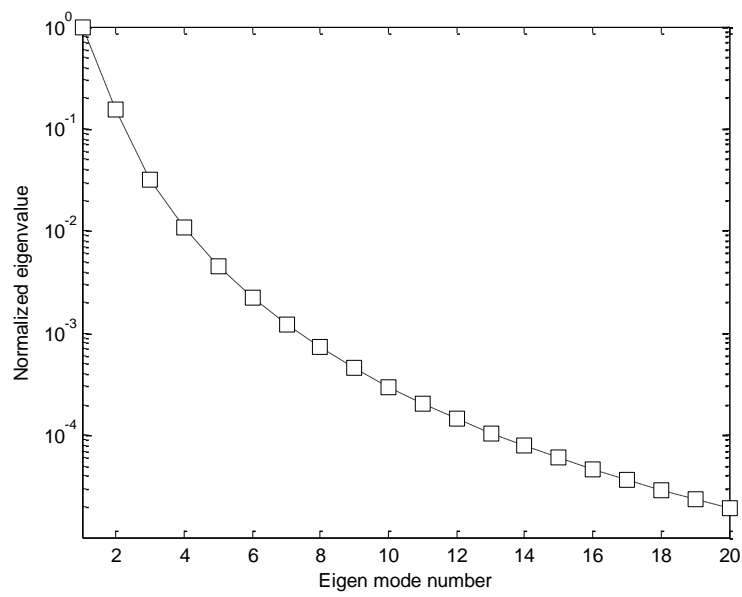

(a)

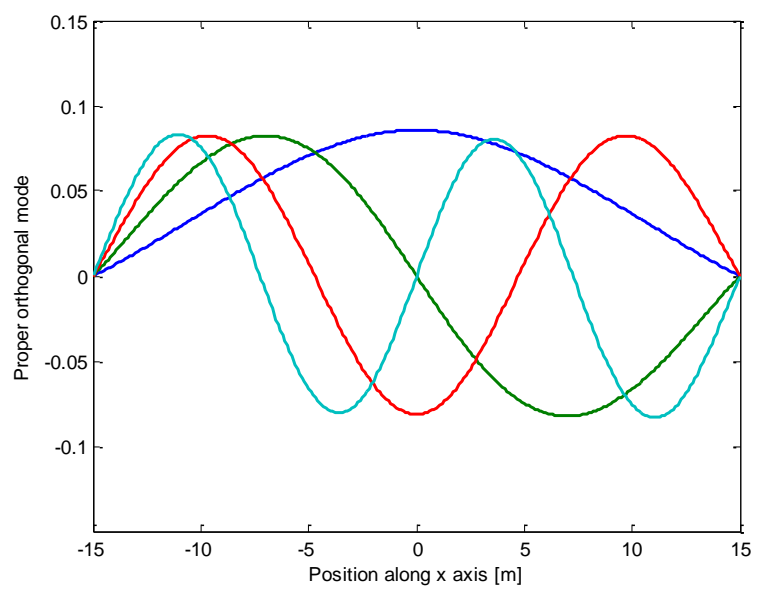

(b)

Fig. 2. Results of POD decomposition a) example of a normalized of singular value b) example of the first proper orthogonal modes.

\section{Benchmark test}

A benchmark test with a particular heat source distribution and time evolution is chosen to test the robustness of the method [8]. Fig. 3a shows the heat source profiles which exhibit a highly heterogeneous field with a slowly time evolution. From these heat source data, the time pattern of the temperature field is derived in the case of two nonconstant, dissymmetric Dirichlet boundary conditions describing the possible warming of the specimen by the grip of the testing machine. A Gaussian white noise of three standard deviations of 50mK representing the noise of the camera was added to the temperature signal. The obtained temperature field is given on Fig. $3 \mathrm{~b}$. The material of the specimen is pure copper which heat diffusivity is dozens of times greater than that of many conventional materials. This thermal characteristic makes the heat source identification even more difficult.

Before carrying out this projection, the temperature field is lifted in order to have a temperature field with boundary conditions equal to zero by superimposing the solution of the thermal problem with zero heat source and boundary conditions equal to the experimental temperature value (Fig. 3c). The temperature field is then projected onto the modes of the reduced POD base (Fig. 3d). The heat source is determined with a finite difference method from the projected temperature. Fig. 3e and Fig. $3 f$ show results obtained with the temperature projected onto respectively 10 and 15 modes. 


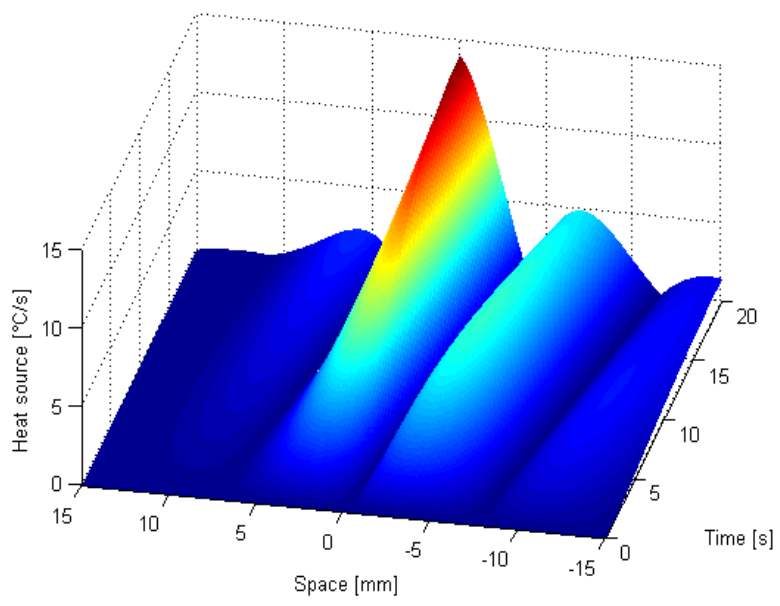

(a)

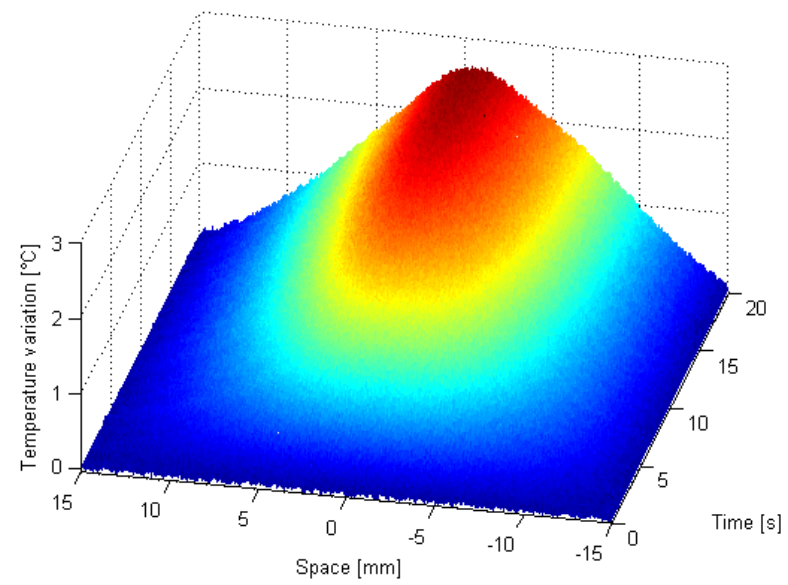

(c)

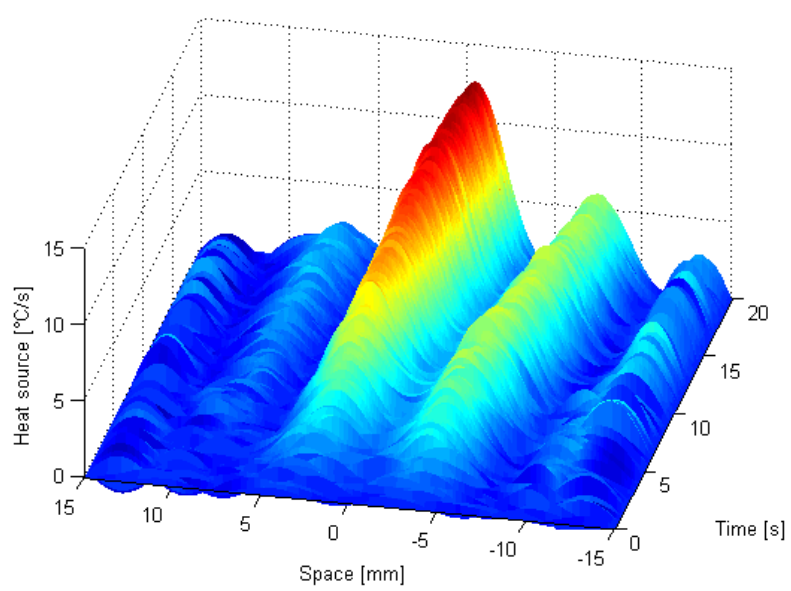

(e)

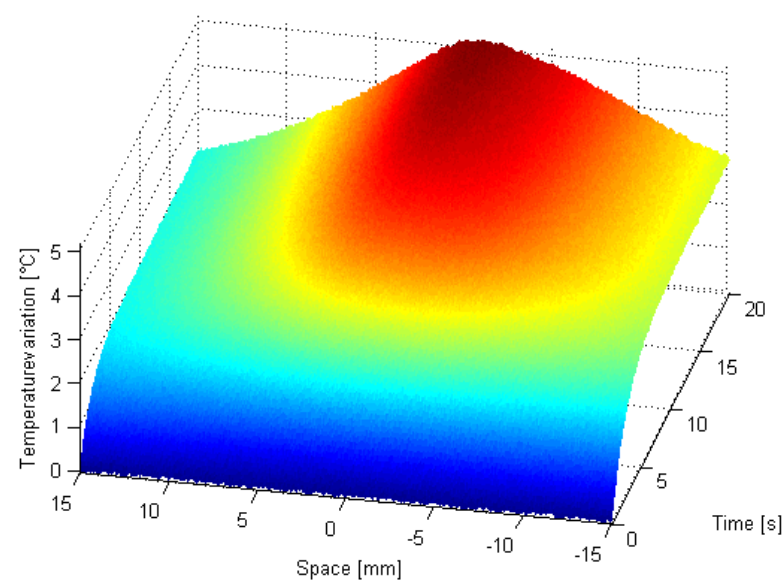

(b)

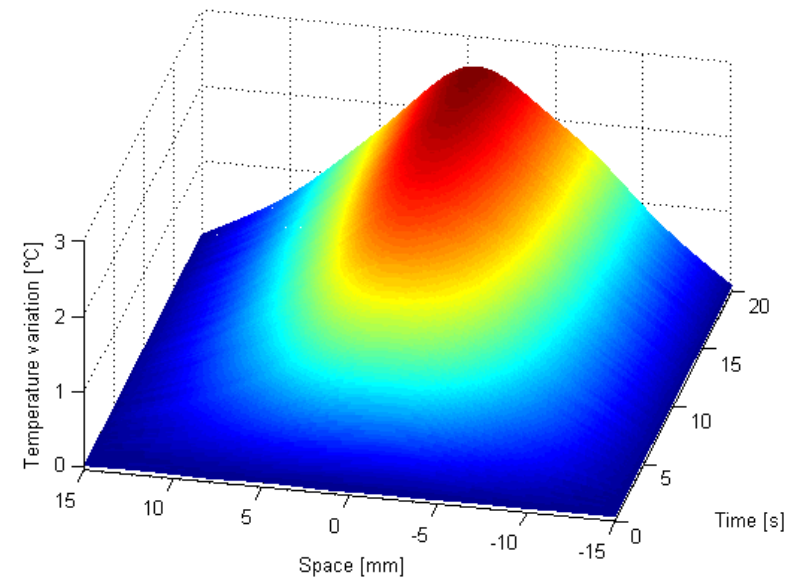

(d)

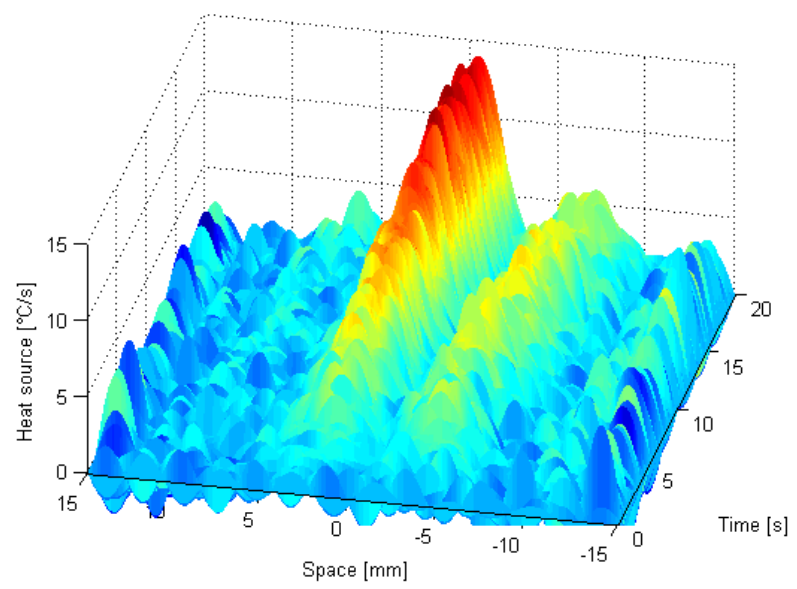

(f)

Fig. 3. Illustration of the various step of $P O D$ method a) reference heat source b) associated noisy temperature field c) temperature field after "lifting" step d) temperature field after projection in the truncated POD base $\left(N_{m}\right)$ e) identification of the heat source with FD method $\left(N_{m}=10\right) f$ ) identification of the heat source with FD method $\left(N_{m}=15\right)$. 
These results show that POD pre-processing coupled with finite differences allows to correctly capture the spatial patterns heterogeneities and the time course of the heat sources. The increase in the number of mode improves for example the estimate of the maximum source. On the other hand it increases in a significant way the noise in particular in the zones where the heat source is weak and exhibit significant gradients (Fig. 4). For example, in the case of a truncated base to $N_{m}=10$ modes, the error on the estimate of the maximum heat source is about $0.83^{\circ} \mathrm{C} / \mathrm{s}$, corresponding to an relative error less than $6 \%$.

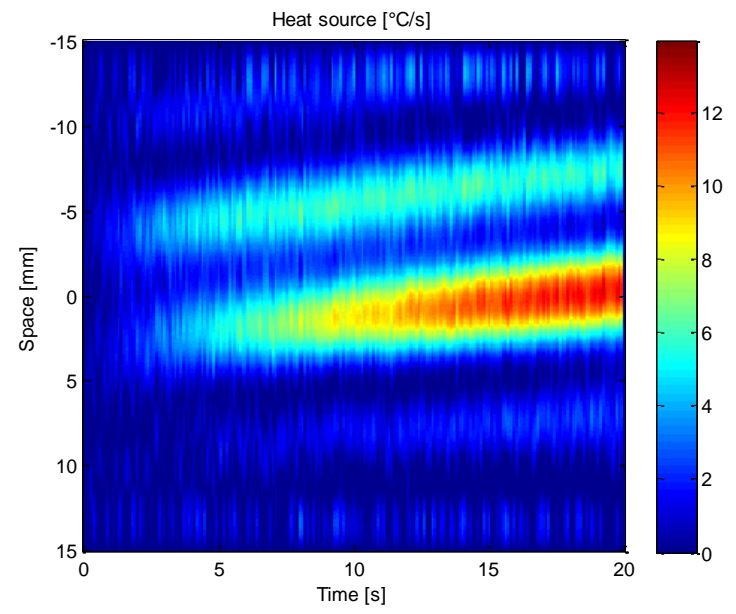

(a)

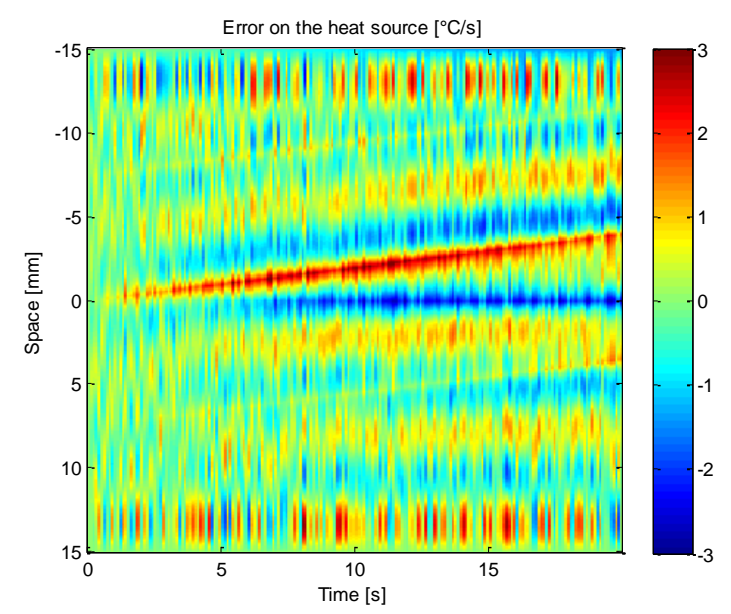

(b)

Fig. 4. Estimate of the error of the heat source identification (number of mode $N_{m}=10$ ) a) reference heat source b) difference between the estimate heat source and the reference heat source.

\section{Comparative analysis of the use of POD before heat source assessment}

Various identification techniques as finite differences (FD), spectral solution (SPS), local least-squares fitting (LSQ) are then applied with and without preprocessing by projection onto the truncated POD base [8]. The number $N_{e f}$ of eigenfunctions chosen to define the truncated spectral solution as well as the number of pixels and frames $\left(2 N_{x}+1\right) \times\left(2 N_{t}+1\right)$ involved in defining the local least-squares approximation and the number of mode used in the truncated POD base are indicated in the column "parameter" of Table 1. To compare the results obtained with the various methods, Table 1 indicates the mean and standard deviation (Std) of the difference between the reference and computed heat sources. The mean value of the reference heat source over the test is about $2.18^{\circ} \mathrm{C} / \mathrm{s}$. $\mathrm{CPU}$ times are also given in the last column of table 1.

Table 1. Heat source assessments using noisy thermal data.

\begin{tabular}{|l|l|l|l|l|}
\hline Method & $\begin{array}{l}\text { Mean } \\
\left(\times 10^{-3}{ }^{\circ} \mathrm{C} / \mathrm{s}\right)\end{array}$ & $\begin{array}{l}\text { Std } \\
\left(\times 10^{-3}{ }^{\circ} \mathrm{C} / \mathrm{s}\right)\end{array}$ & Parameter & $\begin{array}{l}\text { CPU time } \\
(\mathrm{s})\end{array}$ \\
\hline FD & - & - & - & - \\
\hline SPS & -11 & 726 & $\mathrm{~N}_{\mathrm{ef}}=10$ & 37.95 \\
\hline LSQ & 21 & 429 & $\mathrm{~N}_{\mathrm{x}}=15, \mathrm{~N}_{\mathrm{t}}=15$ & 5.37 \\
\hline POD+FD & 1 & 750 & $\mathrm{~N}_{\mathrm{m}}=10$ & $0.52+0.33$ \\
\hline POD+SPS & -12 & 734 & $\mathrm{~N}_{\mathrm{m}}=15, \mathrm{~N}_{\mathrm{ef}}=10$ & $0.61+11.64$ \\
\hline POD+LSQ & -9 & 383 & $\mathrm{~N}_{\mathrm{m}}=15, \mathrm{~N}_{\mathrm{x}}=10, \mathrm{~N}_{\mathrm{t}}=10$ & $0.61+5.34$ \\
\hline
\end{tabular}

The results presented in this table enable us to highlight several points:

- Without POD preprocessing, the FD method gives too noisy estimates of heat sources because of the $2^{\text {nd }}$ order derivative of noisy data that considerably amplifies the noise effects. However, the first result to mention is certainly the fact that the heat sources can be now calculated with the FD method after POD Preprocessing. 
- Regarding the SPS method, the quality of the results is similar to that obtained without POD preprocessing. However this method is really time consuming and the gain in the thermosignal regularity allows a more rapid convergence of the projections whose timing is 3-fold lower for the same number of eigenfunctions.

- $\quad$ Concerning the LSQ method, without POD preprocessing, method the standard deviation is lower than the SPS method because the filtering is made with respect to space and time. Moreover, POD preprocessing slightly improves the standard deviation. The main advantage of this technique is that it allows the reduction of the approximation zone. With the chosen fitting parameters, the size of this approximation zone is 2-fold smaller. As the size of the fitting zone decreases, the fitting function will more precisely describe the local temperature variations and therefore its space and time derivatives and consequently the local heat sources.

\section{Conclusion}

In this paper, the main goal was to show that the use of a truncated POD base of thermal data improves significantly the heat source assessments. A 1D benchmark was thus proposed to test the robustness of the computational methods. Three methods were tested with and without POD preprocessing, the finite difference method, the spectral decomposition and the local least-squares fitting methods. The general advantages of POD preprocessing are as follows:

- $\quad$ The POM are computed once the diffusion problem is defined. They are computed once and for all.

- The number of POM(s) to be considered in the preprocessing depends on the noise characteristic but generally remains small

- The CPU time necessary to project the thermal data are almost negligible compared with those necessary to compute the heat sources

- $\quad$ The POD preprocessing allow to use the FD method with noisy thermal data to calculate heat sources

- $\quad$ Noise reduction by POD enable us to consider a greater number of eigenfunctions for the spectral method and to reduce the fitting zone in the least-square method in order to limit the crushing of thermal gradient for example.

\section{REFERENCES}

[1] Chrysochoos A., "Analyse du comportement thermomécanique des matériaux par thermographie infrarouge", Proceedings of Photomécanique 95, Eyrolles, pp. 203-211, 1995.

[2] Boulanger T, "Analyse par thermographie infrarouge des sources de chaleur induites par la fatigue des aciers", Thèse, Université Montpellier 2, 2004.

[3] Doudard C., Calloch S., Hild F., Roux S., "Identification of heat source fields from infrared thermography: determination of 'self-heating' in a dual-phase steel by using a dog bone sample" Mech. Mat., vol 42, pp.55-62, 2010.

[4] Nayroles B., Bouc R., Caumon H., Chezeau J.C., Giacometti E., "Infrared telethermography and structures mechanics", Int. J. Eng. Sci., vol. 19, pp. 929-947,1981.

[5] Chrysochoos, A. and Louche, H.," An infrared image processing to analyse the calorific effects accompanying strain localisation", Int. J. Eng. Sci., vol.38, pp. 1759-1788, 2000.

[6] Renault N., André S., Maillet D., Cunat C., "A spectral method for the estimation of a thermomechanical heat source from infrared temperature measurements", Int. J. Therm. Sci., vol. 49, pp. 1394-1406, 2010.

[7] Lumley J.L., "The structure of inhomogeneous turbulence". In "Atmospheric Turbulence and Radio Wave Propagation", Nauka Press, Yaglom, Tarasky ed.,pp. 166-178,1967.

[8] Ranc N., Blanche A., Ryckelynck D. , ChrysochoosA. POD preprocessing of IR thermal data to assess heat source. Exp. Mech., In Press, doi:10.1007/s11340-014-9858-2, 2014. 